\title{
The Interictal Continuum: Look Before You Leap
}

\author{
Michael N. Diringer
}

๑ 2018 Springer Science+Business Media, LLC, part of Springer Nature and Neurocritical Care Society

Over the past decade, the use of continuous electroencephalographic (cEEG) monitoring has exploded. It is now commonplace for selected cohorts of patients to routinely undergo a period of cEEG monitoring.

With wider recognition that non-convulsive status epilepticus (NSE) could account for impaired consciousness in critically ill patients, the practice of ordering an EEG to "r/o NSE" became commonplace. Given that these seizures are episodic and often lack a clinical correlate, it is easy to understand how that practice grew into periods of prolonged monitoring and eventually continuous monitoring. Fortuitously, advances in EEG technology have made this option practical to implement and facilitated analysis of the recordings.

The growth in the use of EEG monitoring proved the adage "the harder you look the more you find." Rather than each patient's EEG being sampled for $1-2 \mathrm{~h}$, it was now recorded for $24 \mathrm{~h}$ or more. The higher rate of sampling led to detection of more "abnormalities" that may or may not reflect what we are looking for in the first place (NSE that impairs consciousness). We are presented new findings of uncertain significance that may or may not represent a therapeutic target.

In an attempt to better understand these new findings, we proceed down a well-worn path followed in situations in medicine where improved tools identify new things. First, we seek to categorize and identify meaningful patterns; this results in a period of evolving nosology. Once entities have been defined, the next step, since we are really looking for new therapeutic targets, is to identify relationships between the new entity and outcome. If the new entity is correlated with poor outcome, in our zeal to improve patient care, we skip the next step and look for ways to intervene to treat the entity. This occurs despite having learned many times that doing so can be a fatal mistake. Just because two things are related does not mean the relationship is causal. Usually it is not; many of the entities we identify are related to outcome since they are manifestations of the illness. The worse the disease process, the worse the manifestations and the worse the outcome.

Unfortunately, establishing causality is not so easy, takes a very long time, and in some cases may be impossible. The result is that we are often faced with potential targets for which we think we have interventions. In such situations, it is critical to proceed cautiously, seek signs of effectiveness, and pay careful attention to the risks associated with the intervention.

The interictal continuum (IIC) presents us with such a dilemma. With more widespread and prolonged EEG monitoring, periodic discharges were classified and reclassified and linked to poor outcome. They present a very appealing therapeutic target, and we have a host of interventions. In this issue, Rubinos et al. [1] provide a thoughtful overview of the IIC. They define terminology, review the relationship to outcome, and discuss the rationale for and against treating periodic discharges. They acknowledge that "although these patterns have been well described, we still have little understanding of their pathophysiology and epileptogenic potential, their clinical implication, and how aggressively they should be treated, providing guidance on how to consider whether to treat or not." Given that uncertainty, they propose three creative approaches to treatment that share a common element: They incorporate the use of additional measures to gain additional evidence to support that there is ongoing neuronal injury. In other words, they say look before you leap. 


\section{Source of Support}

There was no funding for this work

Compliance with Ethical Standards

\section{Conflict of interest}

The author declares there are no conflicts of interest.

\section{Reference}

1. Rubinos C, Reynolds AS and Claassen J. The ictal-interictal continuum: to treat or not to treat (and how)? Neurocrit Care (2018). https://doi.org/ 10.1007/s12028-017-0477-5.

Published online: 19 July 2018 